\title{
Interruption of Chemotherapy
}

National Cancer Institute

\section{Source}

National Cancer Institute. Interruption of Chemotherapy. NCI Thesaurus. Code C137910.

Discontinuation of chemotherapy, most often due to toxicity or unacceptable side effects. 